\title{
The Myofibroblastic Conversion of Peribiliary Fibrogenic Cells Distinct from Hepatic Stellate Cells Is Stimulated by Platelet-Derived Growth Factor During Liver Fibrogenesis
}

\author{
Nils Kinnman, Claire Francoz, Véronique Barbu, Dominique Wendum, Colette Rey, \\ Rolf Hultcrantz, Raoul Poupon, and Chantal Housset
}

Institut National de la Santé et de la Recherche Médicale Unité 402, Faculté de Médecine Saint-Antoine (NK, $C F, V B, C R, R P, C H)$, and Service AP-HP d'Hépato-Gastroentérologie (NK, CF, RP, CH) and Service AP-HP d'Anatomie Pathologique (DW), Hôpital Saint-Antoine, and Service AP-HP de Biochimie (CH), Hôpital Tenon, Paris, France; and Department of Gastroenterology and Hepatology (NK, RH), Karolinska Institutet, Karolinska Hospital, Stockholm, Sweden

\begin{abstract}
SUMMARY: The origin of myofibroblasts and the factors promoting their differentiation during liver fibrogenesis remain uncertain. During biliary-type fibrogenesis, the proliferation and chemoattraction of hepatic stellate cells (HSC) toward bile ducts is mediated by platelet-derived growth factor (PDGF), while myofibroblastic conversion of peribiliary cells distinct from HSC also occurs. We herein examined the phenotype of these peribiliary myofibroblasts as compared with myofibroblastic HSC and tested whether their differentiation was affected by PDGF. Biliary-type liver fibrogenesis was induced by common bile duct ligation in rats. After 48 hours, periductular fibrosis in portal tracts colocalized with smooth muscle $\alpha$-actin-immunoreactive myofibroblasts, the majority of which were desmin negative. Simultaneously, in sinusoids, desmin immunoreactivity was induced in a large number of HSC, which were smooth muscle $\alpha$-actin negative. Cultures of peribiliary myofibroblasts were expanded from isolated bile duct segments and compared with myofibroblastic HSC. Peribiliary myofibroblasts outgrowing from bile duct segments expressed smooth muscle $\alpha$-actin, $\alpha 1$ (I) collagen mRNA, and PDGF receptor- $\beta$ subunit. Desmin immunoreactivity gradually decreased in cultured peribiliary myofibroblasts, contrasting with constant labeling of all myofibroblastic HSC. In addition, IL-6 expression in peribiliary myofibroblasts was up to 100-fold lower than in myofibroblastic HSC, whereas the expression of the complement-activating protease P100 in both cell types showed little difference and that of the extracellular matrix component fibulin 2 was similar. The expression of smooth muscle $\alpha$-actin protein in cultured peribiliary myofibroblasts was stimulated by PDGF-BB and inhibited by STI571, a PDGF receptor tyrosine kinase inhibitor, whereas in bile duct-ligated rats, the administration of STI571 caused a significant decrease in peribiliary smooth muscle $\alpha$-actin immunoreactivity, and to a lesser extent, a decrease in peribiliary fibrosis. These results indicate that peribiliary cells distinct from HSC undergo a PDGF-mediated conversion into myofibroblasts expressing IL-6 at lower levels than myofibroblastic HSC and contribute to the initial formation of biliary-type liver fibrosis. (Lab Invest 2003, 83:163-173).
\end{abstract}

$T$ he production of extracellular matrix in the fibrotic liver has been conclusively ascribed to myofibroblast-like cells (Milani et al, 1995) that are considered to derive predominantly from hepatic stellate cells (HSC). A large number of studies have indeed provided evidence that HSC acquire features of a myofibroblastic phenotype after liver injury, what-

\section{DOI: 10.1097/01.LAB.0000054178.01162.E4}

Received October 18, 2002.

This work was supported by grants from the French Association "Vaincre la Mucoviscidose," the Swedish Society of Medicine, the Swedish Society of Medical Research, the Jublin Foundation, the Wennergren foundation, and the Swedish Research Council (9127).

Address reprint requests to: Dr. C. Housset, INSERM U402, Faculté de Médecine Saint-Antoine, 27 rue Chaligny, 75571 Paris Cedex 12, France. E-mail: chantal.housset@st-antoine.inserm.fr ever its cause (Friedman, 2000). In addition, the ability of HSC to differentiate into myofibroblasts has been unambiguously demonstrated in culture models during the past 15 years (de Leeuw et al, 1984). As the role of HSC in liver fibrosis was raising considerable interest, the question of whether myofibroblasts may derive from other cell types in the liver has been generally overlooked. This possibility, nevertheless, has received support from culture studies of isolated human and rat liver cell preparations (Knittel et al, 1999b; Tiggelman et al, 1995; Uchio et al, 2002) and also from histologic analyses of human and rat fibrotic livers (Cassiman et al, 2002; Knittel et al, 1999a). It has been proposed that differences in gene expressions of fibulin 2, an extracellular matrix protein, of P100, a complement-activating protease, and of IL-6 may help distinguish the different origins of myofibroblasts (Knittel et al, 1999b; Tiggelman et al, 1995). 
In biliary-type liver fibrosis induced by bile duct ligation in rats (Kountouras et al, 1984), myofibroblasts accumulate around newly formed bile ducts, in the so-called ductular reaction (Desmet et al, 1995). A dual origin of myofibroblasts in this experimental model has been proposed (Tao et al, 2000). The contribution of HSC is supported by the early myofibroblastic phenotypic changes, which have been identified by analyses of HSC isolated from rat livers at various time points after bile duct ligation. Such changes include increased proliferation and increased expression of platelet-derived growth factor (PDGF) receptor- $\beta$ subunit and of collagen mRNA (Kinnman et al, 2001; Maher and McGuire, 1990; Wong et al, 1994). Furthermore, we recently reported that myofibroblastic HSC undergo chemoattraction toward bile duct structures isolated from bile duct-ligated rats (Kinnman et al, 2000). On the other hand, morphologic studies have attributed the origin of peribiliary myofibroblasts to the phenotypic modulation and proliferation of fibroblasts located in the portal tracts (Tang et al, 1994; Tuchweber et al, 1996; Uchio et al, 2002). Although many investigations have been dedicated to the mechanisms of phenotypic changes in HSC, those effective in locally formed peribiliary myofibroblasts were never examined. Among potential paracrine factors produced by bile duct epithelial cells in the ductular reaction (Grappone et al, 1999; Kinnman et al, 2000), PDGF-BB has the ability not only to stimulate proliferation in mesenchymal cells (Pinzani et al, 1989) but also to trigger a myofibroblastic transdifferentiation of fibrocytes (Oh et al, 1998). In the present study, efforts were continued to define the origin and mechanisms of peribiliary myofibroblast formation in the early stages of biliary-type liver fibrosis. We show that peribiliary cells distinct from HSC give rise to myofibroblasts, both in the intact liver and in culture, and that their myofibroblastic conversion is controlled by PDGF.

\section{Results}

\section{Phenotype of Fibrogenic Cells in Early Biliary-Type Liver Fibrosis}

Biliary-type liver fibrosis was induced by common bile duct ligation in rats. As early as 48 hours after bile duct ligation, an accumulation of extracellular matrix was detected by Sirius red staining around proliferative bile ducts (Fig. 1, A and B). The sinusoids showed a discontinuous staining, which was strictly identical in bile duct-ligated rats and in controls (Fig. 1, A and B). Although smooth muscle $\alpha$-actin ( $\alpha$-SMA) immunolabeling and desmin immunolabeling were restricted to the wall of portal vessels (Fig. 1, C and E) and of terminal hepatic venules (not shown) in control animals, a large number of $\alpha$-SMA-immunoreactive cells that colocalized with fibrosis around bile duct structures were detectable 48 hours after bile duct ligation (Fig. 1D). Sinusoidal $\alpha$-SMA-positive cells were very rare at this stage and strictly confined to the immediate circumference of portal tracts. Simultaneously, by contrast, desmin immunoreactivity was identified in a large number of $\alpha$-SMA-negative sinusoidal cells but only in few periductular cells (Fig. 1F). This analysis supported the view that at this early stage, myofibroblasts causing peribiliary fibrosis did not derive from HSC but from portal fibroblastic cells.

\section{Phenotype of Cultured Peribiliary Myofibroblasts}

We next established cultures of peribiliary myofibroblasts by outgrowth from isolated bile duct segments and compared their phenotype to that of cultureactivated myofibroblastic HSC. Freshly isolated HSC were identified by the fluorescence of their retinoid droplets under UV excitation (Fig. 2, A and B), whereas bile duct preparations were entirely free of HSC contamination as systematically verified by the absence of fluorescence under UV excitation (Fig. 2, C and D). When bile duct segments were cultured in the same conditions as HSC, outgrowth and proliferation of elongated fibroblast-like cells occurred early in culture (Fig. 2C). Within 3 to 4 days in culture, the fibroblastlike cells started to spread, showed cytoplasmic elongations, acquired a myofibroblast-like morphology, and proliferated intensely, whereas round-shaped bile duct epithelial cells detached from the culture plate.

After the first passage, all of the cells expressed vimentin and had a myofibroblast-like appearance. After 4 days in culture and throughout passage 4, all peribiliary myofibroblasts were, like myofibroblastic HSC, $\alpha$-SMA immunoreactive (Fig. 3A). By contrast, desmin immunolabeling, which was evident in approximately $95 \%$ of peribiliary myofibroblasts after 4 days in primary culture, decreased throughout successive passages in culture (Fig. 3B). Eighty percent of peribiliary myofibroblasts were desmin positive at first passage, $60 \%$ at second passage, $50 \%$ at third passage, and approximately $20 \%$ at fourth passage, respectively; $100 \%$ of cultured myofibroblastic HSC were desmin positive throughout passages 1 to 4 .

The fibrogenic potential of peribiliary myofibroblasts was documented by the presence of $\alpha 1$ (I) collagen transcripts (of 4.7 and $5.7 \mathrm{~kb}$ ), which were detected at similar levels in peribiliary myofibroblasts and in myofibroblastic HSC (Fig. 4).

Using real-time PCR, we next examined the expressions of genes that were previously reported to be distinct in HSC and in other liver myofibroblast cell lineages. We found that both peribiliary myofibroblasts and myofibroblastic HSC, irrespective of the passage number, expressed fibulin 2 at high levels (Fig. 5, A and B). Both peribiliary myofibroblasts and, to a lesser extent, myofibroblastic HSC, expressed the complement-activating protease P100 (Fig. 5, A and C), whereas the two cell types clearly showed a different expression of IL-6. A high expression in myofibroblastic HSC contrasted with a low expression in peribiliary myofibroblasts (Fig. 5, A and D). The abundance of IL-6 mRNA was 40- to 100-fold higher in myofibroblastic HSC than in peribiliary myofibroblasts $(p<0.05)$, whereas the differences between fibulin 2 or P100 expressions were not significant. 

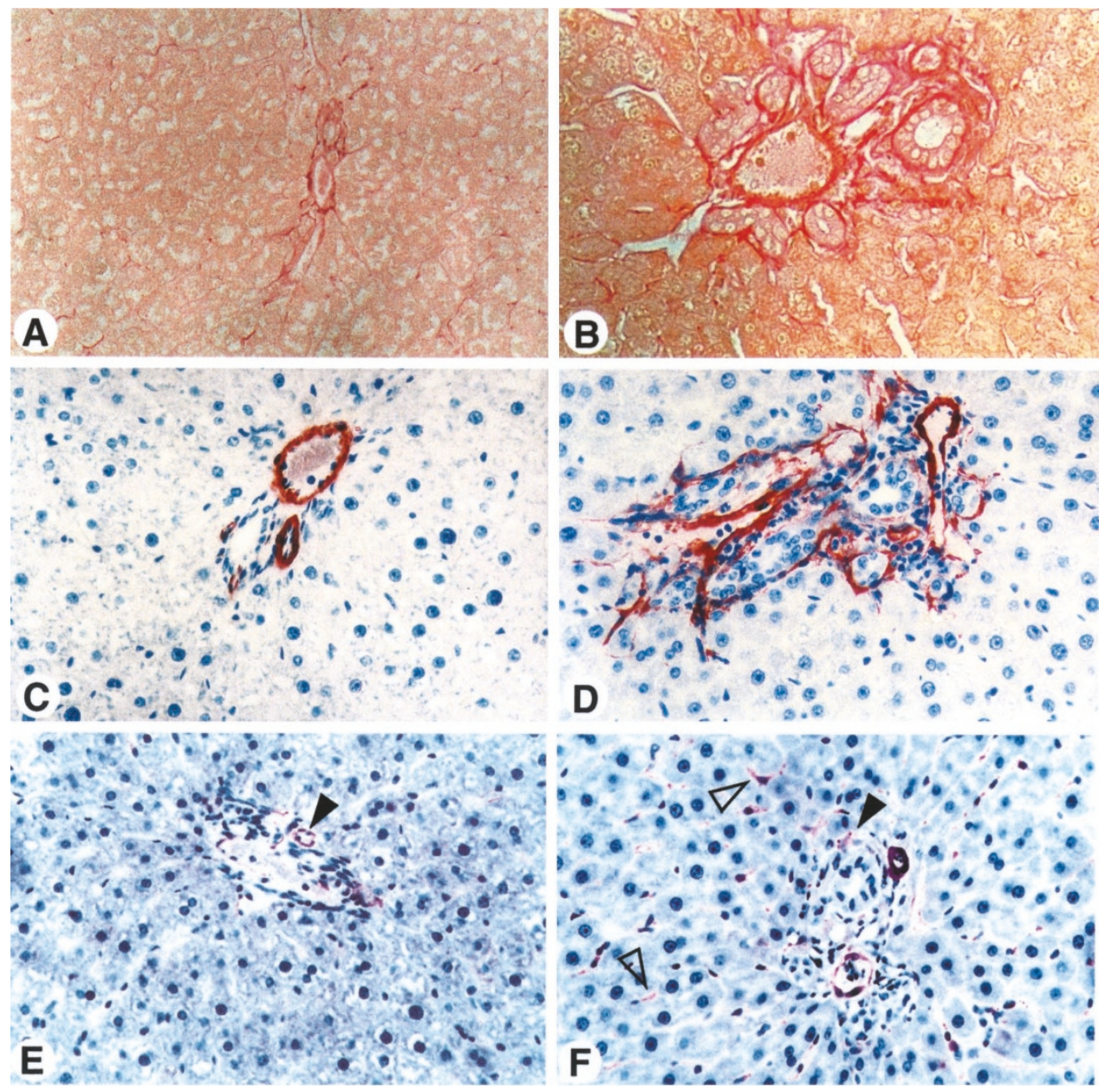

\section{Figure 1.}

Phenotype of fibrogenic cells in early biliary-type liver fibrosis. Liver tissue sections from sham-operated $(A, C$, and $E)$, and bile duct-ligated $(B, D$, and $F)$ rats on postoperative Day 2 were submitted to Sirius red staining (A and B), smooth muscle $\alpha$-actin $(\alpha$-SMA) (C and D), and desmin (E and F) immunolabeling. Portal tract-centered photomicrographs are shown. In sham-operated rats, collagen staining (A) is detected in low amounts predominantly in portal tracts; $\alpha$-SMA (C) and desmin (E) (arrowhead) immunoreactivity is restricted to portal vessel walls. In bile duct-ligated rats, proliferative bile ducts are encircled by fibrosis (B) and by $\alpha$-SMA immunoreactivity (D) restricted to the portal tracts and to rare immediately adjacent sinusoidal cells, whereas desmin immunoreactivity $(\mathrm{F})$ is detected in a large number of sinusoidal cells (open arrowheads) and in only rare periductular cells (closed arrowhead). Original magnifications: A, $\times 200 ; B$ to $F, \times 400$.

\section{Effect of PDGF in the Formation of Peribiliary Myofibroblasts}

PDGF receptor- $\beta$ immunolabeling was evident in peribiliary myofibroblasts as early as Day 4 in primary culture (Fig. 6A). After the first passage and beyond, $100 \%$ of peribiliary myofibroblasts, similar to myofibroblastic HSC, expressed PDGF receptor- $\beta$ (Fig. 6B).

The early induction of PDGF receptor- $\beta$ expression indicated that it could be a triggering event in the activation of peribiliary myofibroblasts, a possibility that was further explored both in vitro and in vivo. The incubation of peribiliary myofibroblasts in primary culture with PDGF-BB caused an increase in the expression of $\alpha$-SMA protein (Fig. 7). This stimulating effect was completely abrogated by simultaneous incubation of the cells with STI571, a protein-tyrosine kinase inhibitor that shows potent and selective inhibition of PDGF-mediated events (Buchdunger et al, 1996, 2000). Furthermore, STI571 partly inhibited the expression of $\alpha$-SMA induced by 5\% FCS (Fig. 7).
In vivo the expression of $\alpha$-SMA was reassessed after the administration of STI571. In bile duct-ligated rats receiving daily treatment with STI571, the number of bile duct structures was unchanged, whereas a substantial decrease in the number of $\alpha$-SMA-positive peribiliary cells was observed. The remaining $\alpha$-SMA reactivity after STI571 treatment was mainly accounted for by vessel walls (Fig. 8, A and B). Semiquantitative morphometric analyses showed that in STI571-treated rats, the surface occupied by $\alpha$-SMAimmunolabeled cells was $1.89 \pm 0.14 \%$ as compared with $3.41 \pm 0.38 \%$ in rats receiving vehicle alone $(p<$ 0.05). Furthermore, morphometric analyses of Sirius red staining in the same rats showed that the fibrotic areas were reduced from $3.16 \pm 0.39 \%$ in controls to $2.16 \pm 0.10 \%$ in STI571-treated animals $(p<0.05)$.

\section{Discussion}

The present study adds to the evidence that cell lineages distinct from HSC contribute to liver fibrogen- 

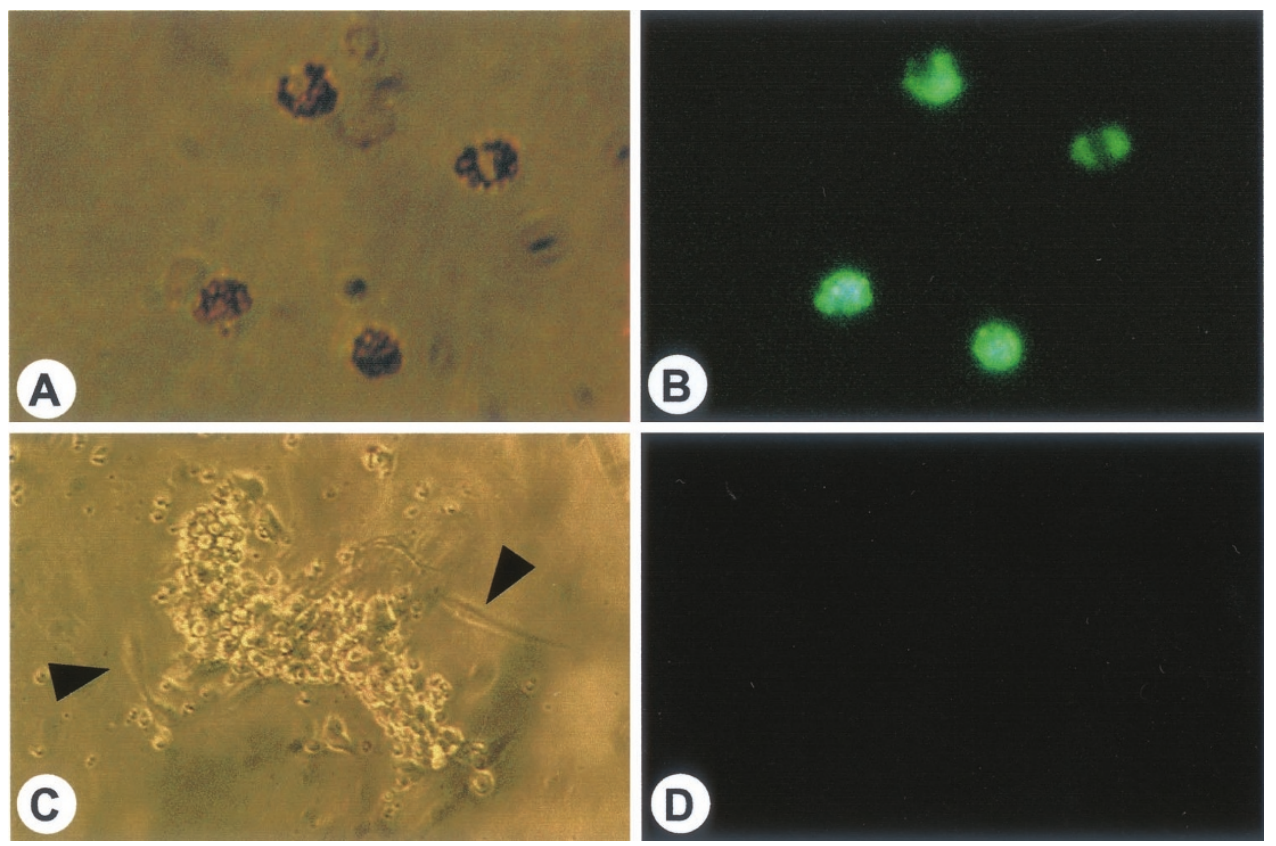

Figure 2.

Hepatic stellate cells (HSC) and bile duct segments are mutually exclusive in cell isolates. Freshly isolated HSC identified (A) under light microscopy, exhibit (B) a characteristic autofluorescence under UV excitation at 328-nm wavelength. Bile duct segment 24 hours after plating shows fibroblast-like cells outgrowing from the periphery (arrowheads) (C) under light microscopy and no fluorescence (D) under UV excitation, demonstrating the absence of HSC in the preparation. Original magnification: $A$ to $D, \times 400$.
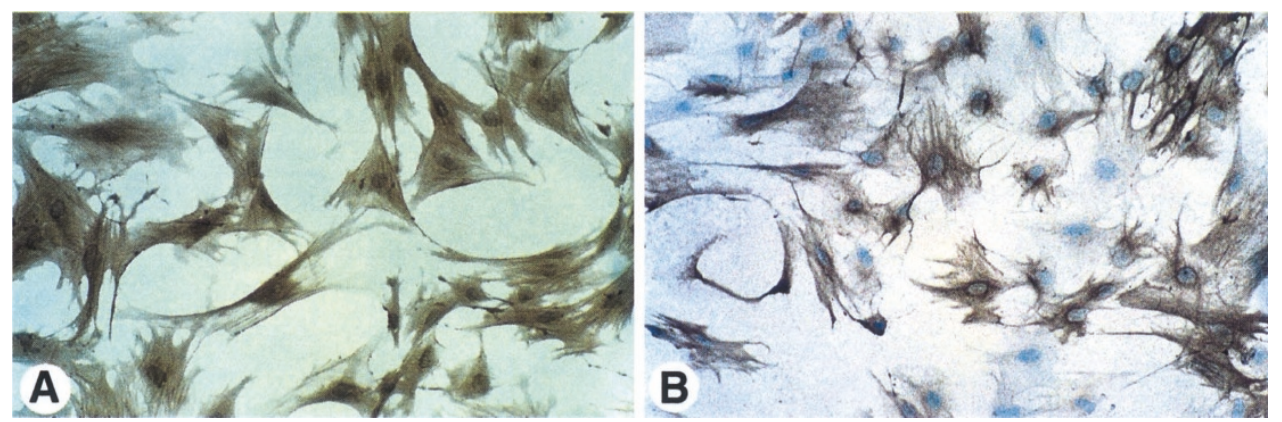

Figure 3.

Expression patterns of cytoskeletal proteins in cultured peribiliary myofibroblasts. Cultures of peribiliary myofibroblasts were established by outgrowth from bile duct segments and submitted to an avidin-biotin immunoperoxidase method. A, A homogeneous pattern of $\alpha$-SMA expression. B, A heterogeneous pattern of desmin expression in subcultured cells (passage 3 ). Original magnification, $\times 400$.

esis. In bile duct-ligated rats, initial accumulation of fibrosis in portal tracts coincided with that of peribiliary myofibroblasts, in which a high expression of $\alpha$-SMA contrasted with inconstant desmin expression. The phenotype of peribiliary myofibroblasts at this stage was clearly different from that of HSC, showing a marked increase in desmin expression, a lack of $\alpha$-SMA expression, and no evidence of fibrosis deposition in sinusoids. The use of paraffin-embedded tissue sections rather than cryosections explains why sinusoidal desmin immunoreactivity was virtually undetectable in normal livers in the present study, as opposed to others (Cassiman et al, 2002). However, the method sensitivity allowed us to confirm that a marked increase in the intensity of sinusoidal desmin immunoreactivity is an early feature of liver injury, as noted before (Geerts et al, 1991; Kinnman et al, 2001).
It was also previously shown that desmin immunoreactivity around bile ducts subsequently increases at later stages of biliary fibrosis in bile duct-ligated animals (Grinko et al, 1995; Hines et al, 1993; Tuchweber et al, 1996), making it more difficult to decipher the origin of myofibroblasts at these later stages. The migration of HSC to portal tracts in particular is likely to be more efficient at later time points, because the migratory capacity of HSC augments during their conversion into myofibroblasts (lkeda et al, 1999). The portal origin of peribiliary myofibroblasts has been further documented both in the present and a previous study (Uchio et al, 2002) by the in vitro emergence of myofibroblasts from bile duct preparations totally devoid of HSC. These peribiliary cells in culture, like culture-activated HSC, exhibit typical features of a myofibroblastic phenotype including the expression of 


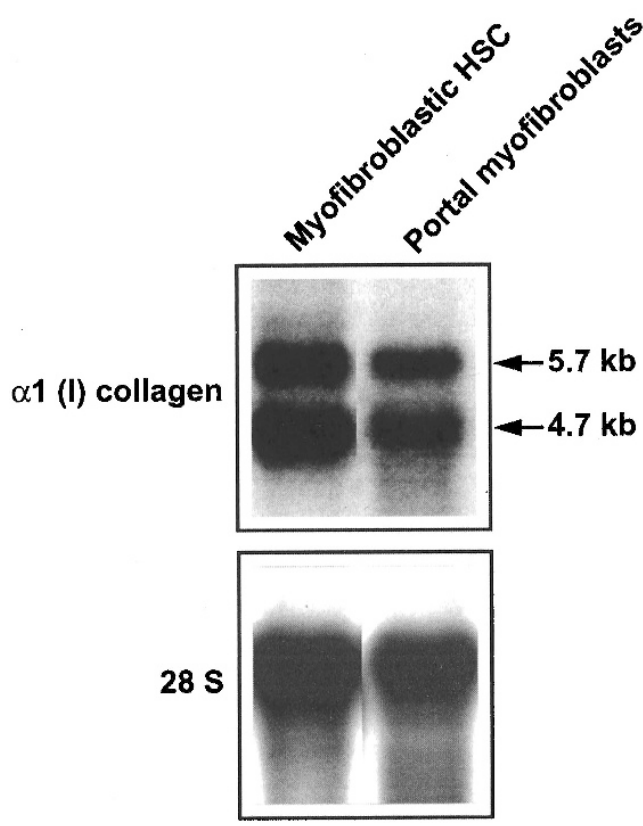

Figure 4.

RNA blot analysis of type I collagen expression in peribiliary myofibroblasts and myofibroblastic HSC. Total RNA $(5 \mu \mathrm{g})$ from peribiliary myofibroblasts and from myofibroblastic HSC (both at passage 4) was subjected to electrophoresis and transfer to membranes. RNA blots were hybridized successively with $\alpha 1$ (I) collagen cDNA and $28 \mathrm{~S}$ oligonucleotide ${ }^{32}$ P-labeled probes. A representative autoradiogram is shown.

$\alpha$-SMA, of $\alpha 1$ (I) collagen, and of the PDGF receptor- $\beta$. However, as compared with culture-activated myofibroblastic HSC, they display differences in the expression of desmin and IL-6. Although the expression of desmin is an early and constant feature of myofibroblastic HSC, its distribution among peribiliary myofibroblasts is heterogeneous and the proportion of desmin-positive cells gradually decreases in subculture. This finding might reflect a heterogeneity in the peribiliary myofibroblast population, as noted in vivo and in other culture models of rat liver myofibroblasts (Knittel et al, 1999b). It should be stressed that a heterogeneity of the myofibroblastic population with regard to desmin, $\alpha$-SMA, and vimentin, the three filaments most often used to classify myofibroblasts, has clearly been shown also in other organs (Kohnen et al, 1996; Schmitt-Graff et al, 1994). However, the possibility is raised that peribiliary myofibroblasts at least partly derive from vascular smooth muscle cells of portal tract vessels, because smooth muscle cells lose desmin immunolabeling after the first passage in culture (Takase et al, 1988).

The phenotype of peribiliary myofibroblasts was further characterized by the detection of fibulin 2, P100, and IL-6. Although Knittel et al (1999b) reported a differential expression of fibulin 2 in HSC as opposed to another population of rat liver myofibroblasts, we found that it was expressed at similar levels in peribiliary myofibroblasts and in myofibroblastic HSC. Also, although in the report by Knittel et al (1999b), P100 was present in HSC but at the limit of detection in rat liver myofibroblasts, we found that the expression of P100 in peribiliary myofibroblasts and myofibroblastic HSC was similar, even with a trend toward a higher expression in peribiliary myofibroblasts. Another common feature between peribiliary myofibroblasts and HSC that was recently reported is the expression of cellular retinol-binding protein-1, a protein involved in vitamin A metabolism (Uchio et al, 2002). Of interest, however, we found that the steadystate levels of IL-6 mRNA were noticeably lower in peribiliary myofibroblasts than in myofibroblastic HSC, which confirms and expands previous findings in cultures of human liver myofibroblasts (Tiggelman et al, 1995). In cultures of this latter cell type, vimentin but not desmin was expressed and the production of IL-6 was consistently (5- to 6-fold) lower than in cultures of human HSC. Given the crucial role of IL-6 in liver regeneration (Cressman et al, 1996), we may postulate that HSC compared with other myofibroblasts ensure different functions during liver wound healing.

In the present study, we also show that PDGF-BB stimulates the expression of $\alpha$-SMA, the hallmark of myofibroblastic differentiation, in the peribiliary cells. Accordingly, when the PDGF receptor downstream signaling cascade was blocked by STI571 in bile duct-ligated rats, $\alpha$-SMA immunoreactivity was dramatically reduced. The decrease in the number of $\alpha$-SMA-positive peribiliary myofibroblasts in STI571treated rats was accompanied by a significant, although less dramatic, reduction in collagen deposition around bile ducts. These results suggest that the production of extracellular matrix and the myofibroblastic differentiation in peribiliary cells are regulated by overlapping but distinct mechanisms. A better understanding of these mechanisms is needed to better delineate the relative contributions of peribiliary myofibroblasts and of HSC to fibrogenesis and to other aspects of liver wound repair.

\section{Materials and Methods}

\section{Animal Model}

All experiments were performed in compliance with the national ethical guidelines for the care and use of laboratory animals. Biliary-type liver injury was induced in male Sprague-Dawley rats ( $R$ Janvier, Le Genest St. Isle, France) weighing 200 to $250 \mathrm{gm}$, by double ligation and section of the common bile duct (Kountouras et al, 1984). Sham-operation consisted of laparotomy and exposure of the common bile duct. To examine the influence of PDGF in peribiliary myofibroblast formation, a group of rats were treated with STI571, an inhibitor of PDGF receptor tyrosine kinase (provided by Dr. Elisabeth Buchdunger, Novartis Pharma Inc., Basel, Switzerland) (Buchdunger et al, 1996, 2000). Daily ip injections of STI571 (20 mg/kg) dissolved in $\mathrm{Me}_{2} \mathrm{SO}$ and diluted 1:20 ( $\left.\mathrm{vol} / \mathrm{vol}\right)$ in PBS began 1 day before bile duct ligation. Vehicle alone was injected in control bile duct-ligated rats and sham-operated rats. Rats were killed and the liver was processed for histologic analyses on postoperative Day 2. Each group consisted of five rats. 

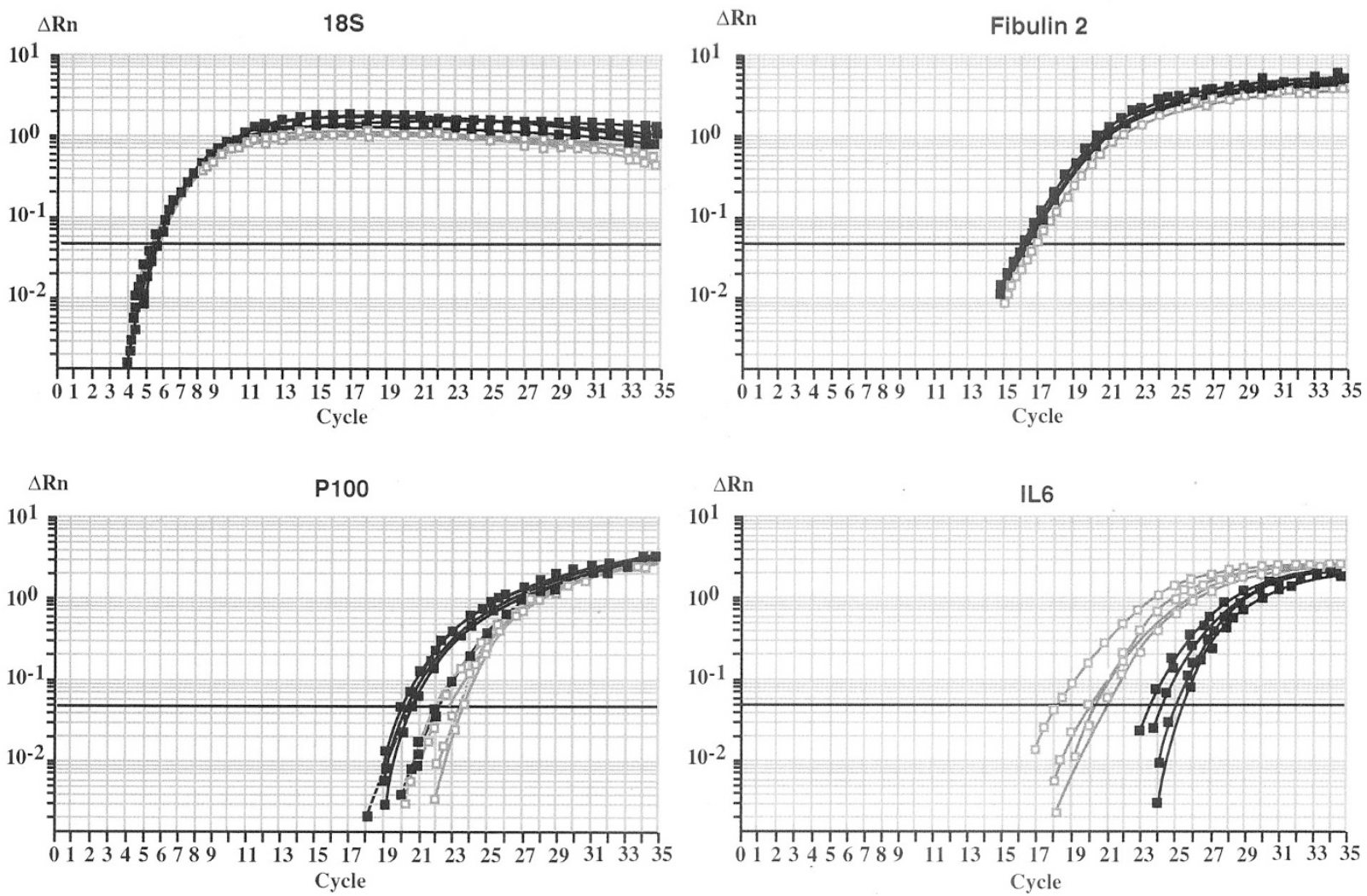

B Fibulin 2

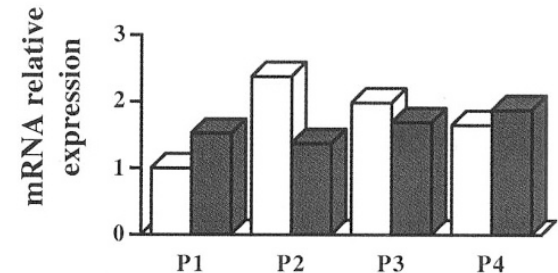

C

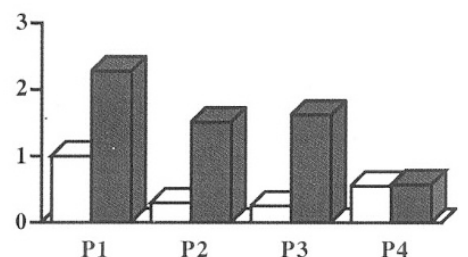
P100
D

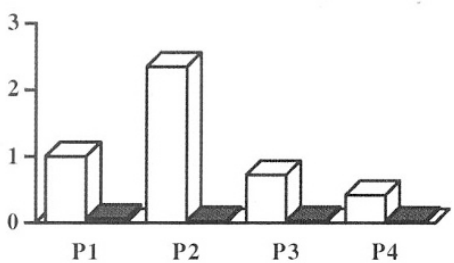

Figure 5.

Real-time PCR analysis of fibulin 2, P100, and IL-6 expression in peribiliary myofibroblasts and in myofibroblastic HSC. Total RNA (1 $\mu$ g) from peribiliary myofibroblasts (closed symbols and bars) and from myofibroblastic HSC (open symbols and bars) at passages 1 to 4 (P1-P4) was reversed transcribed. Real-time PCR of target and 18S cDNA fragments was performed. A, Normalized emission-fluorescence $(\Delta \mathrm{Rn})$ is plotted against cycle number. Threshold is shown. Ct value is determined as the cycle number at which the curve crosses the threshold. B to D, The data shown in A are expressed as the relative amounts of target transcripts in each sample, using myofibroblastic HSC at P1 as a control.

\section{Histologic Analyses}

Serial $4-\mu \mathrm{m}$-thick tissue sections of formalin-fixed, paraffin-embedded liver tissue were stained with hematoxylin-eosin and picrosirius red or submitted to immunohistochemical analyses as follows. Tissue sections were first incubated with an anti-desmin or anti- $\alpha$-SMA mouse mAb (both from Dako S.A., Trappes, France) diluted 1:20 and 1:100, respectively, for 30 minutes at room temperature. After washing in
Tris buffer solution, $\mathrm{pH} 7.6$, tissue sections were incubated successively with peroxidase-conjugated rabbit anti-mouse and swine anti-rabbit polyclonal Abs (Dako S.A.), for 40 minutes at room temperature. Peroxidase activity was revealed by 3-amino-9ethylcarbazole (Vector Laboratories, Burlingame, California). The slides were counterstained with Mayer's hematoxylin and mounted in Glycergel aqueous mounting medium (Dako S.A.). In negative controls, 


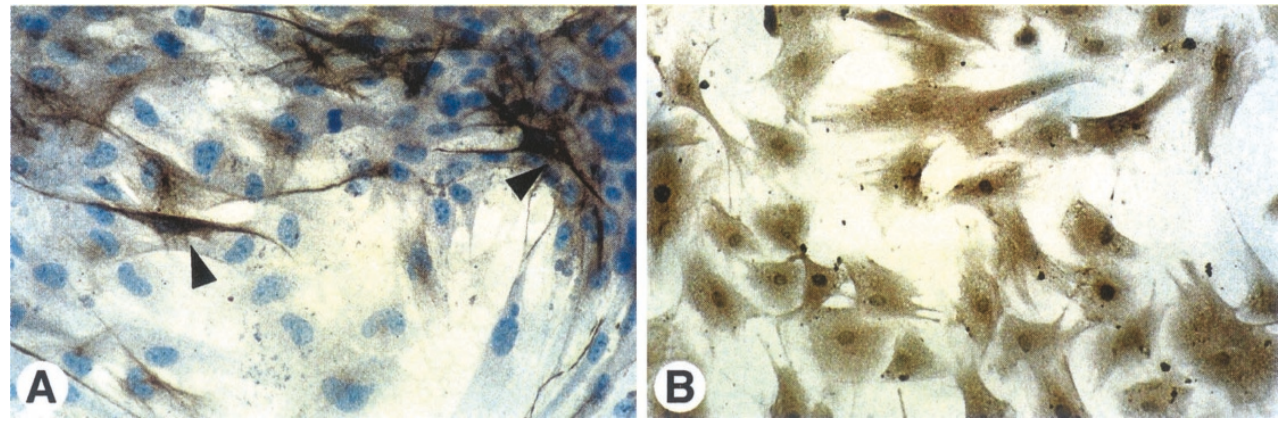

Figure 6 .

Expression pattern of platelet-derived growth factor (PDGF) receptor- $\beta$ subunit in cultured peribiliary myofibroblasts. PDGF receptor- $\beta$ was detected by an avidin-biotin immunoperoxidase method (A) in most of the peribiliary cells with a myofibroblastic appearance (arrowheads) in primary culture (Day 4) and (B) in all subcultured cells (passage 3 ). Original magnification, $\times 400$.

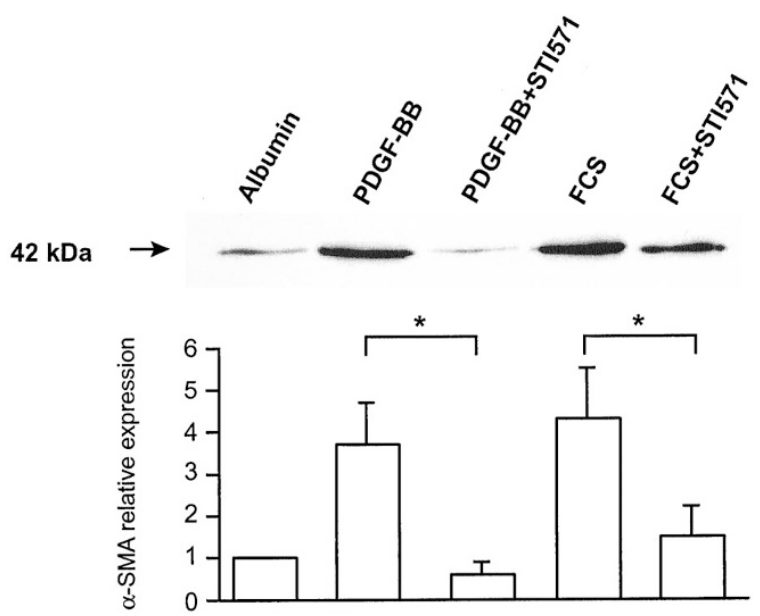

Figure 7.

Immunoblot analysis of $\alpha$-SMA expression in cultured peribiliary myofibroblasts. Peribiliary myofibroblasts in primary culture were incubated for 72 hours with $0.2 \%$ albumin, PDGF-BB $(10 \mathrm{ng} / \mathrm{ml})$, PDGF-BB $(10 \mathrm{ng} / \mathrm{ml}) \mathrm{com}$ bined with STI571 (5 nmol/ml), 5\% FCS, or 5\% FCS combined with STI571 (5 $\mathrm{nmol} / \mathrm{ml})$, and then subjected to immunoblot-ECL analysis of $\alpha$-SMA. A representative blot and the quantitative data (means \pm SEM) of four separate experiments are shown. ${ }^{*} p<0.05$

the primary $A b$ was either omitted or replaced by irrelevant mouse Abs (Dako S.A.). Sirius red staining and $\alpha$-SMA immunolabeling were quantified using a computerized image analysis system (Easy Image; Bergström Instrument, Solna, Sweden) built around a Nikon Eclipse E800 microscope (Nikon Ltd., Tokyo, Japan). The surface areas of picrosirius red and of $\alpha$-SMA labeling in blinded specimens were measured at a video screen display magnification of $\times 400$ and expressed as the ratio of labeled surface area per total analyzed field surface. The average of the score taken from 10 random fields centered around a portal tract was used to generate a single score for each specimen.

\section{Cell Isolation and Culture}

HSC were isolated from normal rats by an established method (Friedman and Roll, 1987) with modifications (Kinnman et al, 2000). In brief, the liver was perfused in situ successively with $0.18 \%$ pronase and with
0.025\% collagenase B (both from Roche Molecular Biochemicals, Mannheim, Germany). The liver cell suspension was incubated in $0.04 \%$ pronase and 6 $\mathrm{mg} / \mathrm{L}$ deoxyribonuclease (Roche Molecular Biochemicals) under agitation. HSC were then separated by density gradient centrifugation through $8.2 \%$ Nycodenz (Sigma, Saint-Quentin Fallavier, France). The purity of HSC isolates was higher than $98 \%$ as assessed by fluorescence of retinoid-containing vacuoles under UV excitation (Friedman and Roll, 1987; Knook et al, 1982), and cell viability exceeded $90 \%$ as tested by erythrosin exclusion (Krause et al, 1984). HSC isolated from normal rats were grown on uncoated plastic in $75-\mathrm{cm}^{2}$ flasks until confluence in DME (Sigma), containing $4 \mathrm{mmol} / \mathrm{L}$ L-glutamine (GIBCO BRL, Life Technologies, Cergy-Pontoise, France), 100,000 IU-100 mg/L penicillin-streptomycin (GIBCO BRL), 10\% FCS (GIBCO BRL), and 10\% horse serum (GIBCO BRL). The cells were maintained at $37^{\circ} \mathrm{C}$, under $95 \%$ air $/ 5 \% \mathrm{CO}_{2}$. Medium was changed 24 hours after plating and every 48 hours thereafter.

Peribiliary myofibroblasts were isolated by outgrowth from isolated intrahepatic bile duct segments. Intrahepatic bile duct segments were isolated from normal rats, using a method described by Mennone et al (1995), with modifications (Kinnman et al, 2000). In brief, the liver was perfused in situ, at a flow rate of 10 $\mathrm{ml} / \mathrm{min}$, with $0.019 \%$ EDTA in $\mathrm{Ca}^{2+}, \mathrm{Mg}^{2+}$-free Hanks' buffer (GIBCO BRL) for 15 minutes and then with $0.008 \%$ collagenase $\mathrm{B}$ in $\mathrm{Ca}^{2+}, \mathrm{Mg}^{2+}$-containing Hanks' buffer for 10 to 15 minutes. The portal tract residue was mechanically separated from parenchymal tissue, minced, and incubated for 30 minutes under agitation at $37^{\circ} \mathrm{C}$, in minimum essential medium (Sigma) containing $0.066 \%$ collagenase B, $0.055 \%$ pronase, $0.006 \%$ deoxyribonuclease, 3\% FCS, 0.1\% BSA (Sigma), $10 \mathrm{mM} \mathrm{HEPES,} \mathrm{and} \mathrm{100,000}$ IU-100 mg/L penicillin-streptomycin. The suspension was then sequentially filtered through $100-$ and $40-\mu \mathrm{m}$ Nitex Swiss nylon monofilament filters (Becton Dickinson, Le Pont-de-Claix, France). Fragments remaining on the filters were digested in the same solution for 30 minutes and for an additional 30 minutes in a solution of the same composition except that pronase was substituted for hyaluronidase $(0.036 \%$ ) (Roche Molecular 


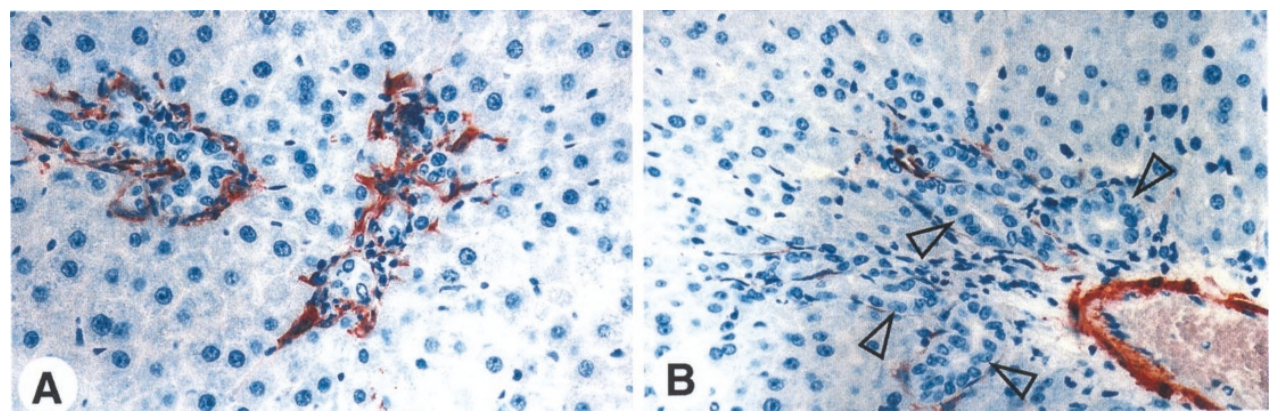

Figure 8.

STI571-induced changes in peribiliary $\alpha$-SMA immunoreactivity in bile duct-ligated rats. Daily ip injections of STI571 (20 mg/kg) or of vehicle were started 1 day before bile duct ligation. $\alpha$-SMA was detected by an indirect immunoperoxidase method on liver tissue sections from (A) vehicle-treated and (B) STI571-treated bile duct-ligated rats on postoperative Day 2. Although in STI571-treated bile duct-ligated rats the number of bile duct structures was unchanged (open arrowheads), periductular $\alpha$-SMA immunoreactivity was dramatically decreased. Representative results obtained in five rats in each group are shown. Original magnification, $\times 400$.

Biochemicals). Fragments were filtered again, and those remaining on the $40-\mu \mathrm{m}$ filter were collected and washed in L15. We have previously demonstrated that more than $90 \%$ of the cells forming the isolated bile duct segments are identified as biliary epithelial cells by cytokeratin 19 immunolabeling and by $\gamma$-glutamyl transpeptidase staining and only display occasional reactivity for $\alpha$-SMA (Kinnman et al, 2000). Bile duct preparations were totally free of contamination by HSC, as proven by the absence of fluorescence under UV excitation. Bile duct segments were grown on plastic in the same culture conditions as HSC. These culture conditions, which promote growth of myofibroblasts, are unfavorable to the survival of biliary epithelial cells. After 5 to 7 days, cells were detached by trypsinization and replated at a 1/4 split ratio. Peribiliary myofibroblasts were then passaged every 7 to 10 days. Experiments were performed in cells in primary culture at Day 4 and in subcultured cells (passages 1-4). To assess the role of PDGF in the activation of peribiliary myofibroblasts in vitro, freshly isolated bile duct segments were plated in 12-well culture plates and allowed to adhere overnight in DME containing $20 \%$ serum (10\% fetal calf/10\% horse). Medium was then changed to DME containing $0.5 \%$ serum $(0.25 \%$ fetal calf $/ 0.25 \%$ horse) for 48 hours. Subsequently, the cells were incubated with 10 $\mathrm{ng} / \mathrm{ml}$ PDGF-BB or DME containing $5 \%$ serum $(2.5 \%$ fetal calf/2.5\% horse), with or without STI571 at a concentration of $5 \mathrm{nmol} / \mathrm{ml}$. Stock solution of 10 $\mathrm{mmol} / \mathrm{L}$ STI571 was prepared in $\mathrm{Me}_{2} \mathrm{SO}$ and stored at $-20^{\circ} \mathrm{C}$. Medium and additives were renewed after 48 hours, and cells were harvested 24 hours later.

\section{Immunoblot}

Immunoblot analysis of $\alpha$-SMA in cultured peribiliary myofibroblasts was performed as previously described (Rockey et al, 1998), with modifications. Cells were washed in PBS and lysed in $50 \mathrm{mM}$ Tris- $\mathrm{HCl}$ containing $0.5 \%$ sodium desoxycholate, $0.1 \%$ SDS, and $1 \%$ Triton and then mixed with a solution containing $62.5 \mathrm{mmol} / \mathrm{L}$ Tris- $\mathrm{HCl}(\mathrm{pH} 6.8), 2 \% \mathrm{SDS}, 10 \%$ glycerol, $0.005 \%$ bromophenol blue, and $5 \%$ 2-mercaptoethanol. Identical amounts of protein ex- tract as determined by Pierce Protein Assay (Interchim, Montluçon, France) were separated by electrophoresis through a $7.5 \%$ SDS-polyacrylamide gel and then transferred to nitrocellulose membranes (Hybond ECL; Amersham Pharmacia Biotech, Orsay, France). Nonspecific binding was blocked by preincubation of the nitrocellulose in Tris buffer solution containing 5\% skim milk overnight at $4^{\circ} \mathrm{C}$. Blots were then incubated with an anti- $\alpha$-SMA mouse mAb (Sigma) diluted $1: 2,000$, for 2 hours at room temperature, and subsequently with peroxidase-conjugated goat anti-mouse IgG (P.A.R.I.S., Compiègne, France) diluted 1:10,000, for 1 hour at room temperature. Immunoreactivity was revealed by enhanced chemiluminescence using an ECL kit (Amersham Pharmacia Biotech). The conditions were established in preliminary experiments to obtain a linear range of response for immunoblotting. The intensities of bands obtained in these conditions were quantified by scanning densitometry.

\section{Immunocytochemistry}

Peribiliary myofibroblasts in primary culture and in subculture and myofibroblastic HSC (passages 1-4) were grown in tissue culture chambers (Becton Dickinson) and then fixed in $100 \%$ acetone at $4^{\circ} \mathrm{C}$ for 10 minutes. Cells were rehydrated in PBS for 10 minutes and then incubated with $1 \%$ normal serum (Vector Laboratories) for 30 minutes. Endogenous biotin was quenched by treatment of the cells with an avidinbiotin blocking kit (SP-2001; Vector Laboratories). The slides were then washed in PBS and incubated with anti- $\alpha$-SMA (1:400) (Sigma), anti-vimentin (1:50), antidesmin (1:20), and anti-PDGF receptor- $\beta$ (5 $\mu \mathrm{g} / \mathrm{ml}$ ) (all from Dako S.A.) primary Abs at room temperature in a moist chamber overnight. After washing in PBS, the slides were incubated with biotin-conjugated secondary Abs diluted 1:200 for 30 minutes, followed by incubation with avidin-biotin-peroxidase complex (Vectastain ABC-HP-kit; Vector Laboratories) for 30 minutes. Color reaction was developed by incubation with diaminobenzidine. The slides were counterstained with Mayer's hematoxylin and mounted in Glycergel aqueous mounting medium. In negative controls, the primary Abs were either omitted or re- 
placed by irrelevant isotype-matched Abs (Vector Laboratories).

\section{Northern Blot}

Total RNA was extracted from cultured cells (passages 1-4) using the Qiagen RNA/DNA kit (Qiagen, Courtaboeuf, France), subjected to electrophoresis on a denaturing $1 \%$ agarose formaldehyde gel, and subsequently transferred to a nylon membrane (Hybond $\mathrm{N}$; Amersham Pharmacia Biotech). After prehybridization, the membranes were incubated with ${ }^{32} \mathrm{P}$-labeled probes $\left(10^{6} \mathrm{cpm} / \mathrm{ml}\right)$ in $50 \%$ deionized formamide, $6 \times$ SSPE (GIBCO BRL), $0.1 \% \quad \mathrm{Na}_{4} \mathrm{P}_{2} \mathrm{O}_{7} \cdot 10 \mathrm{H}_{2} \mathrm{O}, 0.1 \%$ SDS, $5 \times$ Denhardt' s solution, and $0.5 \mathrm{mg} / \mathrm{ml}$ heparin, for 16 hours at $42^{\circ} \mathrm{C}$. Based on the published sequence of rat $\alpha 1$ (I) collagen (Brandsten et al, 1999), cDNA probes were prepared from a PCR product obtained from amplification with the following primers: 5'CAG AGG TTT CAG TGG TTT 3' (sense) and 5' ATT GGC ACC TIT AGC ACC 3' (antisense). 28S oligonucleotide probe was used as an internal standard (Barbu and Dautry, 1989). After stringent posthybridization wash, membranes were processed for autoradiography.

\section{Reverse-Transcription and Real-Time PCR}

Total RNA was extracted from cultured cells using Trizol lysis solution (GIBCO BRL), according to the method of Chomczynski and Sacchi (1987). Firststrand cDNA was generated from $1 \mu \mathrm{g}$ of total RNA using $200 \mathrm{U}$ of Moloney murine leukemia virus reverse transcriptase (Life Technologies) and $5 \mu \mathrm{mol} / \mathrm{L} \mathrm{pd}(\mathrm{N})_{6}$ primers (Pharmacia Biotech). The following primers were designed according to published rat cDNA sequences of P100, fibulin 2, and IL-6 in the GenBank database, using the Primer Express software v1.5 (PE Applied Biosystems, Foster City, California): P100, forward 5'gTTCCgggTCCAgCTTTACTT3', reverse 5'TTgCCAgCACCTggTCTTC3'; fibulin 2, forward 5'gTATCCCTgCAgCggTCTgT3', reverse 5' ATCTTggCCAggAAggTggT3'; and IL-6, forward 5'TGGAGTTCCGTTTCTACCTGGA3', reverse 5'ATGAACTAGGGTTTCAGTATTGCTCTG3'. The following primers were previously designed to amplify a 18S cDNA fragment used as an internal reference: 18S, forward 5'GAGCGAAAGCATTTGCCAAG3', reverse 5'GGCATCGTITATGGTCGGAA3' (Corpechot et al, 2002). A scale from $1.25 \mathrm{pg}$ to $1.25 \times 10^{5} \mathrm{pg}$ of a calibrated total RNA (PE Applied Biosystems) was reverse transcribed with the Moloney murine leukemia virus reverse transcriptase and $\operatorname{pd}(\mathrm{N})_{6}$ primers and used as a standard. Quantitative real-time PCR was performed using the Sybr Green PCR Core Reagents Kit (PE Applied Biosystems) on a PE Applied Biosystems 7700 Sequence Detector (Morrison et al, 1998). Because Sybr Green I binds to any doublestrand DNA, optimization of all PCR reactions was performed to eliminate any amplification product at 35 cycles in the absence of cDNA matrix. Real-time PCR reactions were run in duplicate in $25 \mu$ l with the Sybr Green PCR Core Reagents containing uracyl-N- glycosylase and at concentrations of $\mathrm{MgCl}^{2}$ and dNTPs suggested by the manufacturer. In all reactions, 200 $\mathrm{nmol} / \mathrm{L}$ of each target forward and reverse primers and $50 \mathrm{nM}$ of each $18 \mathrm{~S}$ forward and reverse primers were used. The amplification conditions on the PE Applied Biosystems 7700 sequence detector were the following: 2 minutes at $50^{\circ} \mathrm{C}, 10$ minutes at $95^{\circ} \mathrm{C}, 35$ cycles of $95^{\circ} \mathrm{C} 15$ seconds and $60^{\circ} \mathrm{C} 1$ minute for all reactions. Data were collected and analyzed with Sequence Detector v1.7 software (PE Biosystems). The average efficiency of all PCR reactions given by the calibration curves was $90 \%$. Emission fluorescence was measured at each cycle and, in each sample, normalized by the emission intensity of an internal reference dye, to generate a $\Delta \mathrm{Rn}$ value. The $\mathrm{Ct}$ (threshold cycle) value was determined as the cycle number at which fluorescence first rises above threshold, and a standard curve of $18 \mathrm{~S}$ Ct values obtained from calibrated RNA was established. Data were expressed as the relative amounts of target gene transcripts in each sample, using myofibroblastic HSC P1 as a control, according to the formula $2^{-[\Delta C t \text { sample - } \Delta \text { Ct control] }}$ (Pfaffl, 2001). In each sample, the relative quantification of target gene transcripts was calculated in comparison to a reference gene (18S), as $\Delta \mathrm{Ct}=$ target gene $\mathrm{Ct}-18 \mathrm{~S} \mathrm{Ct}$.

\section{Statistical Analysis}

Data are presented as mean \pm SEM. In the calculation of mean values and statistical variation, "n" refers to the number of separate experiments. Differences between groups were analyzed by using the unpaired Student's $t$ test. A p value less than 0.05 was considered as significant.

\section{References}

Barbu V and Dautry F (1989). Northern blot normalization with a 28S rRNA oligonucleotide probe. Nucleic Acids Res 17: 7115.

Brandsten C, Lundmark C, Christersson C, Hammarstrom L, and Wurtz T (1999). Expression of collagen alpha1(I) mRNA variants during tooth and bone formation in the rat. $\mathrm{J}$ Dent Res 78:11-19.

Buchdunger E, Cioffi CL, Law N, Stover D, Ohno-Jones S, Druker BJ, and Lydon NB (2000). Abl protein-tyrosine kinase inhibitor STI571 inhibits in vitro signal transduction mediated by c-Kit and platelet-derived growth factor receptors. J Pharmacol Exp Ther 295:139-145.

Buchdunger E, Zimmermann J, Mett H, Meyer T, Muller M, Druker BJ, and Lydon NB (1996). Inhibition of the Abl protein-tyrosine kinase in vitro and in vivo by a 2-phenylaminopyrimidine derivative. Cancer Res 56:100104.

Cassiman D, Libbrecht L, Desmet V, Denef C, and Roskams T (2002). Hepatic stellate cell/myofibroblast subpopulations in fibrotic human and rat livers. J Hepatol 36:200-209.

Chomczynski P and Sacchi N (1987). Single-step method of RNA isolation by acid guanidinium thiocyanate-phenolchloroform extraction. Anal Biochem 162:156-159.

Corpechot C, Barbu V, Wendum D, Kinnman N, Rey C, Poupon R, Housset C, and Rosmorduc O (2002). Hypoxia- 
induced VEGF and collagen I expressions are associated with angiogenesis and fibrogenesis in experimental cirrhosis. Hepatology 35:1010-1021.

Cressman DE, Greenbaum LE, DeAngelis RA, Ciliberto G, Furth EE, Poli V, and Taub R (1996). Liver failure and defective hepatocyte regeneration in interleukin-6-deficient mice. Science 274:1379-1383.

de Leeuw AM, McCarthy SP, Geerts A, and Knook DL (1984). Purified rat liver fat-storing cells in culture divide and contain collagen. Hepatology 4:392-403.

Desmet V, Roskams T, and Van Eyken P (1995). Ductular reaction in the liver. Pathol Res Pract 191:513-524.

Friedman SL (2000). Molecular regulation of hepatic fibrosis, an integrated cellular response to tissue injury. J Biol Chem 275:2247-2250.

Friedman SL and Roll FR (1987). Isolation and culture of hepatic lipocytes, Kupffer cells, and sinusoidal endothelial cells by density gradient centrifugation with Stractan. Anal Biochem 161:207-218.

Geerts A, Lazou JM, De Bleser P, and Wisse E (1991). Tissue distribution, quantitation and proliferation kinetics of fatstoring cells in carbon tetrachloride-injured rat liver. Hepatology 13:1193-1202.

Grappone C, Pinzani M, Parola M, Pellegrini G, Caligiuri A, DeFranco R, Marra F, Herbst H, Alpini G, and Milani S (1999). Expression of platelet-derived growth factor in newly formed cholangiocytes during experimental biliary fibrosis in rats. J Hepatol 31:100-109.

Grinko I, Geerts A, and Wisse E (1995). Experimental biliary fibrosis correlates with increased numbers of fat-storing and Kupffer cells, and portal endotoxemia. J Hepatol 23:449458.

Hines JE, Johnson SJ, and Burt AD (1993). In vivo responses of macrophages and perisinusoidal cells to cholestatic liver injury. Am J Pathol 142:511-518.

Ikeda K, Wakahara T, Wang YQ, Kadoya H, Kawada N, and Kaneda K (1999). In vitro migratory potential of rat quiescent hepatic stellate cells and its augmentation by cell activation. Hepatology 29:1760-1767.

Kinnman N, Goria O, Wendum D, Gendron M-C, Rey C, Poupon R, and Housset C (2001). Hepatic stellate proliferation is an early platelet-derived growth factor-mediated cellular event in rat cholestatic liver injury. Lab Invest 81:17091716.

Kinnman N, Hultcrantz R, Barbu V, Rey C, Wendum D, Poupon R, and Housset C (2000). PDGF-mediated chemoattraction of hepatic stellate cells by bile duct segments in cholestatic liver injury. Lab Invest 80:697-707.

Knittel T, Kobold D, Piscaglia F, Saile B, Neubauer K, Mehde M, Timpl R, and Ramadori G (1999a). Localization of liver myofibroblasts and hepatic stellate cells in normal and diseased rat livers: Distinct roles of (myo-)fibroblast subpopulations in hepatic tissue repair. Histochem Cell Biol 112:387401.

Knittel T, Kobold D, Saile B, Grundmann A, Neubauer K, Piscaglia F, and Ramadori G (1999b). Rat liver myofibroblasts and hepatic stellate cells: Different cell populations of the fibroblast lineage with fibrogenic potential. Gastroenterology 117:1205-1221.
Knook DL, Seffelaar AM, and de Leeuw AM (1982). Fatstoring cells of the rat liver: Their isolation and purification. Exp Cell Res 139:468-471.

Kohnen G, Kertschanska S, Demir R, and Kaufmann P (1996). Placental villous stroma as a model system for myofibroblast differentiation. Histochem Cell Biol 105:415-429.

Kountouras J, Billing BH, and Scheuer PJ (1984). Prolonged bile duct obstruction: A new experimental model for cirrhosis in the rat. Br J Exp Pathol 65:305-311.

Krause AW, Carley WW, and Webb WW (1984). Fluorescent erythrosin $B$ is preferable to trypan blue as a vital exclusion dye for mammalian cells in monolayer culture. J Histochem Cytochem 32:1084-1090.

Maher JJ and McGuire RF (1990). Extracellular matrix gene expression increases preferentially in rat lipocytes and sinusoidal endothelial cells during hepatic fibrosis in vivo. J Clin Invest 86:1641-1648.

Mennone A, Alvaro D, Cho W, and Boyer JL (1995). Isolation of small polarized bile duct units. Proc Natl Acad Sci USA 92:6527-6531.

Milani S, Herbst H, Schuppan D, Grappone C, and Heinrichs OE (1995). Cellular sources of extracellular matrix proteins in normal and fibrotic liver: Studies of gene expression by in situ hybridization. J Hepatol 22:71-76.

Morrison TB, Weis JJ, and Wittwer CT (1998). Quantification of low-copy transcripts by continuous SYBR Green I monitoring during amplification. Biotechniques 24:954$958,960,962$.

Oh SJ, Kurz H, Christ B, and Wilting J (1998). Platelet-derived growth factor- $\beta$ induces transformation of fibrocytes into spindle-shaped myofibroblasts in vivo. Histochem Cell Biol 109:349-357.

Pfaffl MW (2001). A new mathematical model for relative quantification in real-time RT-PCR. Nucleic Acids Res 29: 2003-2007.

Pinzani M, Gesualdo L, Sabbah GM, and Abboud HE (1989). Effects of platelet-derived growth factor and other polypeptide mitogens on DNA synthesis and growth of cultured rat liver fat-storing cells. J Clin Invest 84:1786-1793.

Rockey DC, Fouassier L, Chung JJ, Carayon A, Vallee P, Rey C, and Housset C (1998). Cellular localization of endothelin-1 and increased production in liver injury in the rat: Potential for autocrine and paracrine effects on stellate cells. Hepatology $27: 472-480$.

Schmitt-Graff A, Desmouliere A, and Gabbiani G (1994). Heterogeneity of myofibroblast phenotypic features: An example of fibroblastic cell plasticity. Virchows Arch 425:3-24.

Takase S, Leo MA, Nouchi T, and Lieber CS (1988). Desmin distinguishes cultured fat-storing cells from myofibroblasts, smooth muscle cells and fibroblasts in the rat. J Hepatol 6:267-276.

Tang L, Tanaka Y, Marumo F, and Sato C (1994). Phenotypic change in portal fibroblasts in biliary fibrosis. Liver 14:76-82.

Tao LH, Enzan H, Hayashi Y, Miyazaki E, Saibara T, Hiroi M, Toi M, Kuroda N, Naruse K, Jin YL, and Guo LM (2000). Appearance of denuded hepatic stellate cells and their subsequent myofibroblast-like transformation during the early stage of biliary fibrosis in the rat. Med Electron Microsc 33:217-230. 
Tiggelman AM, Boers W, Linthorst C, Brand HS, Sala M, and Chamuleau RA (1995). Interleukin-6 production by human liver (myo)fibroblasts in culture: Evidence for a regulatory role of LPS, IL-1 beta and TNF alpha. J Hepatol 23:295-306.

Tuchweber B, Desmouliere A, Bochaton-Piallat ML, RubbiaBrandt L, and Gabbiani G (1996). Proliferation and phenotypic modulation of portal fibroblasts in the early stages of cholestatic fibrosis in the rat. Lab Invest 74:265-278.

Uchio K, Tuchweber B, Manabe N, Gabbiani G, Rosenbaum $\mathrm{J}$, and Desmouliere A (2002). Cellular retinol-binding protein-1 expression and modulation during in vivo and in vitro myofibroblastic differentiation of rat hepatic stellate cells and portal fibroblasts. Lab Invest 82:619-628.
Wong L, Yamasaki G, Johnson RJ, and Friedman SL (1994). Induction of $\beta$-platelet-derived growth factor receptor in rat hepatic lipocytes during cellular activation in vivo and in culture. J Clin Invest 94:1563-1569. 